\title{
Admissibility of Uncertain Injections in Quadratic Algebraic Systems
}

\author{
Cong Wang, Student Member, IEEE, Eleni Stai, Member, IEEE, and Jean-Yves Le Boudec, Fellow, IEEE
}

\begin{abstract}
We study the admissibility problem in multivariate algebraic systems such as AC electrical networks, where the power injection is quadratic in the state. The goal of such systems is to ensure that the state stays in some security set (e.g., magnitudes of nodal voltages and branch currents are within safety bounds). A common practice is to implicitly control the state by controlling the injection; a difficulty is that the number of states that correspond to a given injection can be zero or many. Further, the injection is subject to some uncertainty. The admissibility problem is whether it is possible to ensure that the state stays in the security set, given that the only available information is some uncertainty set that constrains the injection. We extend the recently proposed V-control theory, design a solution framework to test if a given uncertainty set is admissible, and develop a concrete method for AC electrical networks.
\end{abstract}

Index Terms-control, multivariate quadratic algebraic systems, security constraints, uncertainty, non-singularity, polynomial optimization, convex relaxation, feasibility check.

\section{INTRODUCTION}

In multivariate industrial systems like AC electrical networks and pipeline networks of incompressible flows, the system injection is a quadratic algebraic function of the system state. For example, in an AC electrical network, the system injection (represented by real and imaginary parts of all complex nodal powers) is determined by the system state (represented by real and imaginary parts of all complex nodal voltages) via the quadratic power-flow function.

In such systems, due to the underlying physical nature, the state usually evolves as a continuous function of continuous time. During the evolution, the system's goal is to ensure that the state stays in some predefined security set $\mathcal{V}^{\text {secure }}$ where (i) every state in $\mathcal{V}^{\text {secure }}$ satisfies the system security constraints, and (ii) the Jacobian matrix of the quadratic algebraic system is non-singular at every state in $\mathcal{V}^{\text {secure }}$. For this goal, a common practice is to implicitly control the state by explicitly controlling the injection. For example, in electrical networks, this is conducted by means of power-electronic devices.

Due to uncertainties, the actual implemented injection might differ from its target value, and is often considered to reside in some known uncertainty set, $\mathcal{S}^{\text {uncertain }}$. This makes it difficult to ensure that the actual achieved state stays in $\mathcal{V}^{\text {secure }}$, and hence may result in malfunction of the system. Motivated by this issue, the "admissibility problem" is formulated as: whether it is possible to guarantee that the state stays in $\mathcal{V}^{\text {secure }}$, given that the only available information is that the injection stays in $\mathcal{S}^{\text {uncertain }}$. More specifically, given an initial state

This work is supported by SNSF-NRP70 "Energy Turnaround".

The authors are with École Polytechnique Fédérale de Lausanne (EPFL), CH-1015 Lausanne, Switzerland (e-mail: cong.wang@epfl.ch; eleni.stai@epfl.ch; jean-yves.leboudec@epfl.ch). $\mathbf{v}^{\text {initial }} \in \mathcal{V}^{\text {secure }}$ and given a set $\mathcal{S}^{\text {uncertain }}$ that contains the initial injection, we say that $\mathcal{S}^{\text {uncertain }}$ is admissible for $\mathbf{v}^{\text {initial }}$ if any continuous path of the state, such that the corresponding injection remains in $\mathcal{S}^{\text {uncertain }}$, must stay in $\mathcal{V}^{\text {secure }}$ (a formal definition is presented in Definition 1 of Section II-A).

For this problem, the main difficulty is that every injection in $\mathcal{S}^{\text {uncertain }}$ can have zero or multiple corresponding states, owing to the quadratic nature of the multivariate function that maps state to injection. In [1], it has been shown that it is not sufficient that every injection in $\mathcal{S}^{\text {uncertain }}$ has at least one corresponding state in $\mathcal{V}^{\text {secure }}$ In such cases, the state can escape $\mathcal{V}^{\text {secure }}$ (see Figure 1 in [1]). In contrast [1, Lemma $2]$, the problem goes away if every injection in $\mathcal{S}^{\text {uncertain }}$ has exactly one corresponding state in $\mathcal{V}^{\text {secure }}$ (and given the condition that $\mathcal{V}^{\text {secure }}$ is open and non-singular). However, as we show in Section II-D, this condition is impractical, since there are scenarios in which it is possible to ensure that the state stays in $\mathcal{V}^{\text {secure }}$, though there are injections in $\mathcal{S}^{\text {uncertain }}$ that have multiple corresponding states in $\mathcal{V}^{\text {secure }}$.

To solve the admissibility problem, our approach consists in constructing an appropriate set of states $\mathcal{V}$ such that $\mathcal{V}$ is a subset of $\mathcal{V}^{\text {secure }}$, and such that, for every injection in $\mathcal{S}^{\text {uncertain }}$, there exists a unique corresponding state in $\mathcal{V}$ (by Lemma 2 in [1], this ensures admissibility). To this end, we propose two new theorems (Theorems 2 and 3 in Section III-A) that guarantee such existence and uniqueness. Based on the proposed theorems, we design a solution framework in Section III-B for solving the admissibility problem, and develop a concrete method for AC electrical networks in Section IV. Specifically, our main contributions are as follows:

1) In Section III-B, we propose a framework that uses and extends the results in [1], to solve the admissibility problem. The framework is based on two new theorems in Section III-A:

- In Theorem 2, we show that if a set $\mathcal{V}$ of states is convex and the Jacobian matrix of the system is non-singular everywhere in $\mathcal{V}$, then $\mathcal{V}$ is a domain of uniqueness.

- In Theorem 3, we provide sufficient conditions on an arbitrary set $\mathcal{V}$ of states and on an arbitrary set $\mathcal{S}$ of injections, so that every injection in $\mathcal{S}$ is guaranteed to have a corresponding state in $\mathcal{V}$; the conditions are based on the impossibility of obtaining a state at the boundary of $\mathcal{V}$ for any injection in $\mathcal{S}$.

2) In Sections IV-A and IV-B, we use the proposed framework and develop a concrete method for solving the admissibility problem in AC electrical networks. To evaluate the performance of our concrete method, we apply it to a few test networks. For illustration purposes, in Section V, we show numerical results on a meshed 15-bus CIGRE network and a radial 34-bus IEEE network. 


\section{Problem Formulation and State of the Art}

\section{A. Problem Formulation}

We consider a system where, at any point of time, the state is characterized by vector $\mathbf{v}$ and the injection is vector $\mathbf{s}=\mathbf{F}(\mathbf{v})$. Both $\mathbf{v}, \mathbf{s}$ belong to a Euclidean space of finite dimension $M$, and $\mathbf{F}$ is a quadratic function determined by system parameters. Here, a Euclidean space of dimension $M$ can be either $\mathbb{R}^{M}$ or $\mathbb{C}^{N}$ with $M=2 N$. When the system is represented in $\mathbb{C}^{N}$ with $M=2 N, \mathbf{F}$ is real-quadratic instead of complex-quadratic. We denote the Jacobian matrix of $\mathbf{F}$ evaluated at $\mathbf{v}$ by $\mathbf{J}_{\mathbf{F}}(\mathbf{v})$. For this system, let us study a situation where (i) $\mathbf{v}, \mathbf{s}$ are functions of continuous time $t$, expressed as $\mathbf{v}(t), \mathbf{s}(t)$; (ii) the system is steered by a controller whose goal is to maintain $\mathbf{v}(t)$ in a security set $\mathcal{V}^{\text {secure }}$ that satisfies Assumption 1, by acting on $\mathbf{s}(t)$.

Assumption 1. $\mathcal{V}^{\text {secure }}$ is bounded and described as

$$
\begin{aligned}
& \mathcal{V}^{\text {secure }}=\left\{\mathbf{v}: \mathbf{J}_{\mathbf{F}}(\mathbf{v}) \text { non-singular },\right. \\
& \left.f_{\ell}(\mathbf{v})>0, \ell \in\left\{1, \ldots, L^{\text {secure }}\right\}\right\},
\end{aligned}
$$

where $f_{\ell}$ are continuous functions.

Suppose that, at some time $t_{0}$,

- $\mathbf{v}\left(t_{0}\right)$ is observed and belongs to $\mathcal{V}^{\text {secure }}$;

- The controller decides a new value of injection, $s^{*}$, to be implemented during time interval $\left(t_{0}, t_{1}\right]$ for some $t_{1}>t_{0}$.

Ideally, we expect that $\mathbf{s}(t)=\mathbf{s}^{*}, \forall t \in\left(t_{0}, t_{1}\right]$. But in reality,

- $\mathbf{s}(t)$ is continuous and starts from $\mathbf{s}\left(t_{0}\right)=\mathbf{F}\left(\mathbf{v}\left(t_{0}\right)\right)$;

- $\mathbf{s}(t)$ is uncertain, caused by, e.g., uncaptured dynamics, reaction delays, and disturbances from natural resources.

In a control framework such as Commelec [2], for every value of $\mathbf{s}^{*}$, the controller derives a set of injections $\mathcal{S}^{\text {uncertain }}$ (using the "belief functions"), such that $\mathbf{s}(t) \in \mathcal{S}^{\text {uncertain }}$, $\forall t \in\left[t_{0}, t_{1}\right]$. Due to the uncertainty in $\mathbf{s}(t)$, we cannot precisely know the state, $\mathbf{v}(t)$, for $t \in\left(t_{0}, t_{1}\right]$. This issue leads to a question: given that the only available information of $\mathbf{s}(t)$ is that $\mathbf{s}(t) \in \mathcal{S}^{\text {uncertain }}, \forall t \in\left[t_{0}, t_{1}\right]$, is it possible to ensure that $\mathbf{v}(t) \in \mathcal{V}^{\text {secure }}, \forall t \in\left[t_{0}, t_{1}\right]$ ? We formalize this question as the admissibility problem. Specifically, we assume that $\mathbf{v}(t)$ is continuous and introduce the following concept.

Definition 1. Given any initial state $\mathbf{v}^{\text {initial }}$ and uncertainty set $\mathcal{S}^{\text {uncertain }}$ of injections such that

(I1) $\mathbf{v}^{\text {initial }} \in \mathcal{V}^{\text {secure }}$,

(I2) $\mathbf{F}\left(\mathbf{v}^{\text {initial }}\right) \in \mathcal{S}^{\text {uncertain }}$,

we say $\mathcal{S}^{\text {uncertain }}$ is admissible for $\mathbf{v}^{\text {initial }}$ if, for any continuous function $\mathbf{v}(t), t \in[0,1]$ such that $\mathbf{v}(0)=\mathbf{v}^{\text {initial }}$ and $\mathbf{F}(\mathbf{v}(t)) \in$ $\mathcal{S}^{\text {uncertain }}, \forall t \in[0,1]$, we have that $\mathbf{v}(t) \in \mathcal{V}^{\text {secure }}, \forall t \in[0,1]$.

Using Definition 1, the admissibility problem is as follows.

Admissibility Problem: Given an initial state $\mathbf{v}^{\text {initial }}$ and an uncertainty set $\mathcal{S}^{\text {uncertain }}$ that satisfy conditions (I1)-(I2), is $\mathcal{S}^{\text {uncertain }}$ admissible for $\mathbf{v}^{\text {initial }}$ ?

In other words, the problem is to determine whether the connected component of $\mathbf{F}^{-1}\left(\mathcal{S}^{\text {uncertain }}\right)$ that contains $\mathbf{v}^{\text {initial }}$ is included in $\mathcal{V}^{\text {secure }}$, where $\mathbf{F}^{-1}$ means the pre-image.
B. Application to AC Electrical Networks: Problem Formulation

In this section, we illustrate the problem formulation of Section II-A with the case of an AC electrical network. Later, in Section IV, we will solve the admissibility problem in AC electrical networks using our proposed framework. As we explain in detail below, in this case:

- The state $\mathbf{v}$ is the collection of complex nodal voltages. We use complex values as the formulation is more succinct; this can naturally be mapped to real values using real and imaginary parts (i.e. rectangular coordinates).

- The injection $\mathbf{s}$ is the collection of complex nodal power injections (i.e. active and reactive powers).

- The function $\mathbf{F}$ defines the AC power flow equation (3); it is real quadratic in rectangular coordinates.

- $\mathcal{V}^{\text {secure }}$ is the set of electrical state that satisfy constraints on the magnitudes of branch currents and nodal voltages, (5)-(7).

- $\mathcal{S}^{\text {uncertain }}$ is the set of all possible complex power injections s (see Section $V$ for a numerical example of $\left.\mathcal{S}^{\text {uncertain }}\right)$. Typically, a grid controller gives operational bounds to generators and loads; the set $\mathcal{S}^{\text {uncertain }}$ quantifies the bounds allowed by the controller plus the uncertainty in the implementation of power setpoints [2].

Consider an AC network with one slack bus, $N P Q$ buses and a generic topology (i.e., radial or meshed). We assume that the network topology is fixed and known. In addition, we assign index 0 to the slack bus, and indexes $1, \ldots, N$ to the $P Q$ buses. For convenience of expression, we define

- $\mathcal{N}=\{0, \ldots, N\}$ as the index set of all buses;

- $\mathcal{N}^{P Q}=\mathcal{N} \backslash\{0\}$ as the index set of $P Q$ buses;

- $\mathcal{E}=\{j k:$ a branch exists between buses $j, k \in \mathcal{N}\}$ as a set of ordered index pairs.

At each bus $j \in \mathcal{N}$, we denote the complex nodal voltage, current and power by $v_{j}, i_{j}$ and $s_{j}$, respectively. Furthermore, let $\mathbf{v}=\left(v_{1}, \ldots, v_{N}\right)^{T}, \mathbf{i}=\left(i_{1}, \ldots, i_{N}\right)^{T}$ and $\mathbf{s}=\left(s_{1}, \ldots, s_{N}\right)^{T}$.

By [1], $\mathbf{v}, \mathbf{i}$, and $\mathbf{s}$ fulfill the following relation:

$$
\begin{gathered}
\mathbf{i}=\mathbf{Y}_{L L}(\mathbf{v}-\mathbf{w}), \\
\mathbf{s}=\operatorname{diag}(\mathbf{v}) \overline{\mathbf{i}}
\end{gathered}
$$

where

- $\mathbf{Y}_{L L} \in \mathbb{C}^{N \times N}$ is the invertible ${ }^{1}$ submatrix on the bottomright corner of the $(N+1) \times(N+1)$ nodal admittance matrix, which is characterized by network topology as well as the parameters of $\pi$-modeled transmission lines and transformers [5], [6];

- $\mathbf{w} \in \mathbb{C}^{N}$ is the constant zero-injection state;

- $\operatorname{diag}(\mathbf{v})$ is the diagonal matrix formed by $\mathbf{v}$;

- " - " stands for complex conjugation.

Combine (1) and (2), we have

$$
\begin{aligned}
\mathbf{s} & =\operatorname{diag}(\mathbf{v}) \overline{\mathbf{Y}}_{L L}(\overline{\mathbf{v}}-\overline{\mathbf{w}}) \\
& =\mathbf{F}(\mathbf{v}),
\end{aligned}
$$

which concretely defines the $\mathbf{F}$ in Section II-A.

Now, consider the following.

${ }^{1}$ Detailed proof of invertibility can be found in [3] and [4]. 
- The branch current from bus $j$ to bus $k$ can be expressed as a linear function of $\mathbf{v}$ :

$$
i_{j k}=a_{j k} v_{0}+\mathbf{c}_{j k}^{T} \mathbf{v}
$$

where $a_{j k} \in \mathbb{C}, \mathbf{c}_{j k} \in \mathbb{C}^{N}$ are constants given by the $\pi$-modeled transmission lines and transformers;

- The magnitude of the nodal voltage at bus $j$ should stay within $\left(V_{j}^{\min }, V_{j}^{\max }\right)$, and the magnitude of the branch current from bus $j$ to $k$ should stay in $\left[0, I_{j k}^{\max }\right)$, for some positive real safety bounds $V_{j}^{\min }, V_{j}^{\max }, I_{j k}^{\max }$.

We have the system security constraints as follows:

$$
\begin{gathered}
f_{j}^{\mathrm{V}, \text { low }}(\mathbf{v})=\left|v_{j}\right|^{2}-\left(V_{j}^{\min }\right)^{2}>0, \forall j \in \mathcal{N}^{P Q}, \\
f_{j}^{\mathrm{V}, \text { up }}(\mathbf{v})=-\left|v_{j}\right|^{2}+\left(V_{j}^{\max }\right)^{2}>0, \forall j \in \mathcal{N}^{P Q}, \\
f_{j k}^{\mathrm{I} \text {,branch }}(\mathbf{v})=-\left|a_{j k} v_{0}+\mathbf{c}_{j k}^{T} \mathbf{v}\right|^{2}+\left(I_{j k}^{\max }\right)^{2}>0, \forall j k \in \mathcal{E},
\end{gathered}
$$

which specify the details of the continuous functions $f_{\ell}(\mathbf{v}), \quad \ell \in\left\{1, \ldots, L^{\text {secure }}\right\}$ in Assumption 1 that are used to construct the set $\mathcal{V}^{\text {secure }}$. (Note that in the related work [7], the branch-current constraints (7) are replaced by linear constraints on the angles of $\mathbf{v}$ in polar coordinates, which is invalid in practice.)

\section{State of the Art}

The admissibility problem is a control problem. Different from many existent control problems where the goal is to design a controller that drives the state and injection to some prescribed values (e.g., [8]), the goal of the admissibility problem is to see whether it is possible to guarantee that the state stays in a security set, given that the injection is constrained in some uncertainty set. As aforementioned in Section I, the main difficulty of the problem is that every injection in $\mathbf{S}^{\text {uncertain }}$ can have zero or multiple corresponding states. This difficulty comes from the quadratic nature of the system. ${ }^{2}$ Although upper bounds on the number of corresponding states are given in [9], [10], they do not guarantee uniqueness of the state and hence do not ensure admissibility.

In the literature, the admissibility problem is first investigated in [2] for AC electrical networks. Therein, a solution method is proposed, based on the following assumptions:

- For any injection in $\mathcal{S}^{\text {uncertain }}$, if there exists a corresponding state in $\mathcal{V}^{\text {secure }}$, then this state is unique.

- The extreme values of the state are obtained at the extreme values of the injection.

But these assumptions might not hold in practice. Specifically,

- For an injection, there can be more than one corresponding state in $\mathcal{V}^{\text {secure }}[11]$.

- The extreme values of the state can be obtained at nonextreme values of the injection [1].

In [12], another method is developed for the admissibility problem in AC electrical networks. This method assumes that for every injection in $\mathcal{S}^{\text {uncertain }}$, there exists a corresponding state in $\mathcal{V}^{\text {secure }}$. Based on this assumption, it tests the admissibility by iteratively solving a collection of optimization problems. This method has the following shortcomings:

- An injection may have zero corresponding state in $\mathcal{V}^{\text {secure }}$.

${ }^{2}$ Note that in control problems like [8], only linear system is considered.
- The iterative scheme is heuristic and does not have a guaranteed convergence.

For every injection in $\mathcal{S}^{\text {uncertain }}$ to have at least one corresponding state in $\mathcal{V}^{\text {secure }}$, sufficient conditions are proposed in [7], [13]. These conditions are obtained by exploiting properties (e.g., topology, convexity) in both the space of states and the space of injections. Despite the theoretical merits, these works do not solve the admissibility problem, as the existence of a state in $\mathcal{V}^{\text {secure }}$ for every injection in $\mathcal{S}^{\text {uncertain }}$ is only a necessary condition for the admissibility.

Finally, the works in [14] and [15] propose an approach for repairing convex and polynomial infeasible problems, respectively, by adapting their parameters so that they become solvable. Their focus is to achieve the feasibility of an optimization problem by actually changing it. This is, however, not the target of the admissibility problem, which is a control problem seeking to ensure that the state lies in the security set while injections change over time.

\section{Theory of $\mathcal{V}$-Control}

In this section, we recall some definitions and results from [1] that are originally proposed for AC electrical networks. These results will be enriched in the subsequent sections, in order to solve the admissibility problem.

Definition 2. For a set $\mathcal{V}$ of states and a set $\mathcal{S}$ of injections: $\mathcal{S}$ is a domain of $\mathcal{V}$-control if, for any continuous path $\mathbf{v}(t), t \in$ $[0,1]$ such that $\mathbf{v}(0) \in \mathcal{V}$ and $\mathbf{F}(\mathbf{v}(t)) \in \mathcal{S}, \forall t \in[0,1]$, we have $\mathbf{v}(t) \in \mathcal{V}, \forall t \in[0,1]$.

In Definition 2, the concept of $\mathcal{V}$-control can be interpreted as follows: Ensure the continuous path $\mathbf{v}(t)$ in $\mathcal{V}$ by keeping the continuous path $\mathbf{s}(t)$ in $\mathcal{S}$.

In [1], a number of sufficient conditions are given to guarantee that some set of injections $\mathcal{S}$ is a domain of $\mathcal{V}$ control for some set $\mathcal{V}$. This could be used to address the admissibility problem, if we could show that $\mathcal{S}^{\text {uncertain }}$ is a domain of $\mathcal{V}^{\text {secure }}$-control. However, as we show below, such a direct application might be unsuitable in practice.

Specifically, let us recall the following result from [1], which gives sufficient conditions for $\mathcal{S}$ to be a domain of $\mathcal{V}$-control.

Definition 3. For a set $\mathcal{V}$ of states:

- $\mathcal{V}$ is a domain of uniqueness if $\mathbf{F}(\mathbf{v})=\mathbf{F}\left(\mathbf{v}^{\prime}\right) \Rightarrow \mathbf{v}=$ $\mathbf{v}^{\prime}, \forall \mathbf{v}, \mathbf{v}^{\prime} \in \mathcal{V}$;

- $\mathcal{V}$ is non-singular if $\forall \mathbf{v} \in \mathcal{V}$, the Jacobian matrix $\mathbf{J}_{\mathbf{F}}(\mathbf{v})$ is non-singular.

Theorem 1 (Lemma 2 of [1]). Let $\mathcal{V}$ be a set of states and $\mathcal{S}$ be a set of injections. Assume that

1) $\mathcal{V}$ is open and non-singular;

2) $\forall \mathbf{s} \in \mathcal{S}$, there is a unique $\mathbf{v} \in \mathcal{V}$ such that $\mathbf{F}(\mathbf{v})=\mathbf{s}$.

Then there exists a continuous mapping $\mathbf{G}: \mathcal{S} \rightarrow \mathcal{V}$ such that $\mathbf{F}(\mathbf{G}(\mathbf{s}))=\mathbf{s}, \forall \mathbf{s} \in \mathcal{S}$, and $\mathcal{S}$ is a domain of $\mathcal{V}$-control.

As mentioned above, a direct application of Theorem 1 to the admissibility problem would be showing that $\mathcal{S}^{\text {uncertain }}$ is a domain of $\mathcal{V}^{\text {secure }}$-control. By Assumption 1, a sufficient condition for this $\mathcal{V}^{\text {secure }}$-control to hold would be that every injection in $\mathcal{S}^{\text {uncertain }}$ has exactly one corresponding state in $\mathcal{V}^{\text {secure }}$. However, this could be impractical and unnecessary, 
as there might be some injection in $\mathcal{S}^{\text {uncertain }}$ that corresponds to multiple states in $\mathcal{V}^{\text {secure }}$. To illustrate, let us consider the AC electrical network in Figure 1.

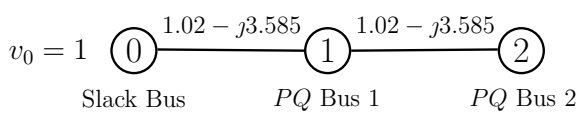

Fig. 1. Network topology with slack-bus voltage and admittances in p.u.

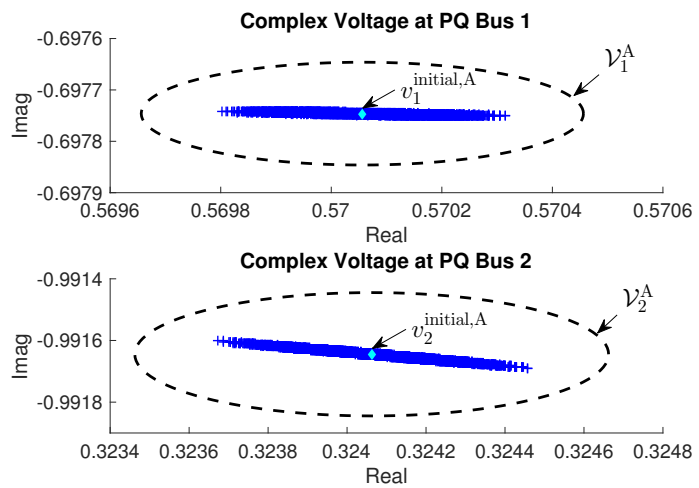

(a)
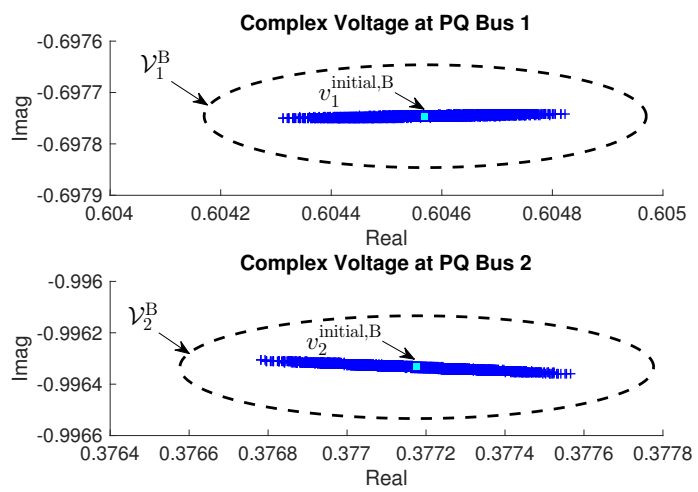

(b)

Fig. 2. (i) Candidate initial states $\left(v_{1}^{\text {initial }, \mathrm{A}}, v_{2}^{\text {initial, } \mathrm{A}}\right)^{T}$ and $\left(v_{1}^{\text {initial }, \mathrm{B}}, v_{2}^{\text {initial, } \mathrm{B}}\right)^{T}$, which are presented by cyan "diamond" and "square"; (ii) All states (marked by scattered blue "+") in $\mathcal{V}^{\text {secure }}$ that correspond to the injections in $\mathcal{S}^{\text {uncertain }}$; (iii) Open and non-singular sets $\mathcal{V}_{1}^{\mathrm{A}} \times \mathcal{V}_{2}^{\mathrm{A}} \subseteq \mathcal{V}^{\text {secure }}$ and $\mathcal{V}_{1}^{\mathrm{B}} \times \mathcal{V}_{2}^{\mathrm{B}} \subseteq \mathcal{V}^{\text {secure }}$.

For ease of exposition, let the complex nodal voltage and the complex nodal power at $P Q$ bus $j \in\{1,2\}$ be $v_{j}, s_{j}$, respectively. As a result, the state is $\mathbf{v}=\left(v_{1}, v_{2}\right)^{T}$ and the injection is $\mathbf{s}=\left(s_{1}, s_{2}\right)^{T}$. Now, suppose that

- A state satisfies the security constraints if the nodal voltage magnitudes at $P Q$ buses are between 0.9-1.1 p.u.;

- The initial injection is set to $s_{1}^{\text {initial }}=-1.105+\jmath 1$ p.u., $s_{2}^{\text {initial }}=-1+\jmath 1.105$ p.u.;

- $\mathcal{S}^{\text {uncertain }}=\left\{\left(s_{1}, s_{2}\right)^{T}:\left|s_{j}-s_{j}^{\text {initial }}\right| \leq 10^{-5}, j \in\{1,2\}\right\}$.

For the given initial injection, the two corresponding states in $\mathcal{V}^{\text {secure }}$ are denoted by $\left(v_{1}^{\text {initial,A }}, v_{2}^{\text {initial, A }}\right)^{T}$ and $\left(v_{1}^{\text {initial,B }}, v_{2}^{\text {initial, } \mathrm{B}}\right)^{T}$. Additionally, every injection in $\mathcal{S}^{\text {uncertain }}$ has two corresponding states in $\mathcal{V}^{\text {secure }}$, marked by scattered "+" in Figure 2. In this example, $\mathcal{S}^{\text {uncertain }}$ is admissible for $\left(v_{1}^{\text {initial, } \mathrm{A}}, v_{2}^{\text {initial, } \mathrm{A}}\right)^{T}$. To see why, construct the open and nonsingular set $\mathcal{V}^{\mathrm{A}}=\mathcal{V}_{1}^{\mathrm{A}} \times \mathcal{V}_{2}^{\mathrm{A}}$ as in Figure 2(a) with

$$
\begin{aligned}
& \text { - } \mathcal{V}_{1}^{\mathrm{A}}=\left\{v_{1}:\left(\frac{\operatorname{Re}\left(v_{1}-v_{1}^{\text {initial }, \mathrm{A}}\right)}{0.0004}\right)^{2}+\left(\frac{\operatorname{Im}\left(v_{1}-v_{1}^{\text {initial }, \mathrm{A}}\right)}{0.0001}\right)^{2}<1\right\} ; \\
& \text { - } \mathcal{V}_{2}^{\mathrm{A}}=\left\{v_{2}:\left(\frac{\operatorname{Re}\left(v_{2}-v_{2}^{\text {intial }, \mathrm{A}}\right)}{0.0006}\right)^{2}+\left(\frac{\operatorname{Im}\left(v_{2}-v_{2}^{\text {initial }, \mathrm{A}}\right)}{0.0002}\right)^{2}<1\right\} .
\end{aligned}
$$

Here, $\operatorname{Re}()$ (resp. $\operatorname{Im}())$ is the real (resp. imaginary) part of a complex value, and " $x$ " means the Cartesian product. Clearly, for each injection in $\mathcal{S}^{\text {uncertain }}$, the corresponding state around $\left(v_{1}^{\text {initial }, \mathrm{A}}, v_{2}^{\text {initial, } \mathrm{A}}\right)^{T}$ is in $\mathcal{V}^{\mathrm{A}}$. Moreover, for each injection in $\mathcal{S}^{\text {uncertain }}$, there is exactly one corresponding state in $\mathcal{V}^{\mathrm{A}}$. Next, by Theorem $1, \mathcal{S}^{\text {uncertain }}$ is a domain of $\mathcal{V}^{\mathrm{A}}$-control and is thus admissible for $\left(v_{1}^{\text {initial, } \mathrm{A}}, v_{2}^{\text {initial, } \mathrm{A}}\right)^{T}$. Note that the same logic can be employed to prove that $\mathcal{S}^{\text {uncertain }}$ is also admissible for $\left(v_{1}^{\text {initial, } \mathrm{B}}, v_{2}^{\text {initial, } \mathrm{B}}\right)^{T}$, using $\mathcal{V}^{\mathrm{B}}=\mathcal{V}_{1}^{\mathrm{B}} \times \mathcal{V}_{2}^{\mathrm{B}}$ in Figure $2(\mathrm{~b})$.

This example illustrates that, in order to apply the theory of $\mathcal{V}$-control in [1] to the admissibility problem, we need to build an appropriate domain $\mathcal{V}$ that is typically smaller than $\mathcal{V}^{\text {secure }}$. We explain how to perform this in the rest of the paper.

\section{THEORETICAL FOUNDATIONS AND SOLUTION FRAMEWORK}

\section{A. Theoretical Foundations}

To solve the admissibility problem, we need new theoretical results that go beyond Theorem 1 . In detail, this is because the 2nd condition in Theorem 1 is difficult to verify in practice. To address this issue, we propose two new theorems that give sufficient conditions for the 2 nd condition in Theorem 1 to hold. Specifically, $\forall \mathbf{s} \in \mathcal{S}$, Theorem 2 gives sufficient conditions for the uniqueness of $\mathbf{v} \in \mathcal{V}$ such that $\mathbf{F}(\mathbf{v})=\mathbf{s}$; Theorem 3 gives sufficient conditions for the existence of $\mathbf{v} \in \mathcal{V}$ such that $\mathbf{F}(\mathbf{v})=\mathbf{s}$.

Theorem 2. If the set $\mathcal{V}$ of states is non-singular and convex, then it is a domain of uniqueness.

Proof. We prove by contradiction. Let set $\mathcal{V}$ be non-singular and convex. In addition, suppose that there exist $\mathbf{v}, \mathbf{v}^{\prime} \in \mathcal{V}$ such that (i) $\mathbf{F}(\mathbf{v})=\mathbf{F}\left(\mathbf{v}^{\prime}\right)$, and (ii) $\mathbf{v} \neq \mathbf{v}^{\prime}$. Owing to convexity, there is $\frac{\mathbf{v}+\mathbf{v}^{\prime}}{2} \in \mathcal{V}$. Furthermore, according to the non-singularity in $\mathcal{V}, \mathbf{J}_{\mathbf{F}}\left(\frac{\mathbf{v}+\mathbf{v}^{\prime}}{2}\right)$ is non-singular. However, by Lemma 1 in Appendix-A, $\mathbf{J}_{\mathbf{F}}\left(\frac{\mathbf{v}+\mathbf{v}^{\prime}}{2}\right)$ should be singular since $\mathbf{F}$ is quadratic. This creates a contradiction.

Theorem 3. Let $\mathcal{V}$ be a set of states, $\mathcal{S}$ be a set of injections, and $\partial \mathcal{V}$ denote the topological boundary of $\mathcal{V}$. Assume that

1) $\mathcal{V}$ is bounded, open and non-singular;

2) $\mathcal{S}$ is connected;

3) $\mathbf{F}(\mathcal{V}) \bigcap \mathcal{S}$ is non-empty;

4) $\mathbf{F}(\partial \mathcal{V}) \bigcap \mathcal{S}$ is empty.

Then, for any $\mathbf{s} \in \mathcal{S}$, there exists $a \mathbf{v} \in \mathcal{V}$ such that $\mathbf{F}(\mathbf{v})=\mathbf{s}$.

In the above, the topological boundary $\partial \mathcal{V}$ of $\mathcal{V}$ is the closed set defined as $\partial \mathcal{V}=\operatorname{cl}(\mathcal{V}) \backslash \operatorname{int}(\mathcal{V})$, where $\operatorname{cl}(\mathcal{V})$ is the closure of $\mathcal{V}$ (i.e., the smallest closed set containing $\mathcal{V}$ ) and $\operatorname{int}(\mathcal{V})$ is the interior of $\mathcal{V}$ (i.e., the largest open set contained in $\mathcal{V}$ ) [16]. It is also the set of points that can be expressed both as the limit of a sequence of points in $\mathcal{V}$ and as the limit of a sequence of points not in $\mathcal{V}$. Furthermore, $\mathcal{S}$ is connected if $\mathcal{S}$ itself and the empty set are the only subsets that are both closed 
and open in $\mathcal{S}$. For $\mathcal{S}$ to be connected, a sufficient condition is that $\mathcal{S}$ is path-connected.

Proof. We need to show that $\mathcal{S} \subseteq \mathbf{F}(\mathcal{V})$, i.e., $\mathbf{F}(\mathcal{V}) \cap \mathcal{S}=\mathcal{S}$. Since $\mathcal{S}$ is connected by condition 2), its closed and open subsets are $\mathcal{S}$ and the empty set. Based on this, consider that (i) $\mathbf{F}(\mathcal{V}) \cap \mathcal{S} \subseteq \mathcal{S}$, and (ii) $\mathbf{F}(\mathcal{V}) \cap \mathcal{S}$ is not empty by condition 3), we can prove $\mathbf{F}(\mathcal{V}) \cap \mathcal{S}=\mathcal{S}$ by showing $\mathbf{F}(\mathcal{V}) \cap \mathcal{S}$ is both closed and open in $\mathcal{S}$, which we do next.

First, the openness of $\mathcal{V}$ implies $\partial \mathcal{V}=\operatorname{cl}(\mathcal{V}) \backslash \mathcal{V}$. As $\mathcal{V}$ is bounded, $\operatorname{cl}(\mathcal{V})$ is compact. By continuity of $\mathbf{F}, \mathbf{F}(\operatorname{cl}(\mathcal{V}))$ is compact and hence closed; thus $\mathbf{F}(\operatorname{cl}(\mathcal{V})) \cap \mathcal{S}$ is closed in $\mathcal{S}$. By condition 4$), \mathbf{F}(\operatorname{cl}(\mathcal{V}) \backslash \mathcal{V}) \cap \mathcal{S}$ is empty. Thus, we have $\mathbf{F}(\operatorname{cl}(\mathcal{V})) \bigcap \mathcal{S}=\mathbf{F}(\mathcal{V}) \cap \mathcal{S}$. So, $\mathbf{F}(\mathcal{V}) \cap \mathcal{S}$ is closed in $\mathcal{S}$.

Second, $\mathcal{V}$ is open and non-singular by condition 1). By the Inverse Function Theorem [17], $\mathbf{F}(\mathcal{V})$ is open and therefore $\mathbf{F}(\mathcal{V}) \cap \mathcal{S}$ is open in $\mathcal{S}$. Thus, $\mathbf{F}(\mathcal{V}) \cap \mathcal{S}$ is a non-empty, closed and open subset in $\mathcal{S}$, which means that $\mathbf{F}(\mathcal{V}) \cap \mathcal{S}=\mathcal{S}$ and completes the proof.

In essence, Theorem 3 asserts that every $\mathrm{s} \in \mathcal{S}$ has a corresponding state in $\mathcal{V}$, provided that

- At least one $\mathbf{s}^{\star} \in \mathcal{S}$ has a corresponding state in $\mathcal{V}$,

- It is impossible for any $\mathbf{s} \in \mathcal{S}$ to have a corresponding state at the boundary of $\mathcal{V}$.

Intuitively, this is because: If there would be an $\mathbf{s}^{\star \star} \in \mathcal{S}$ that has no corresponding state in $\mathcal{V}$, then in order to continuously move from $\mathbf{s}^{\star}$ to $\mathbf{s}^{\star \star}$, the path in the state space must either hit a singular point in $\mathcal{V}$ or exit $\mathcal{V}$ by crossing the boundary $\partial \mathcal{V}$; but this is made impossible by the 1 st and the 4th conditions in Theorem 3.

\section{B. Solution Framework}

Based on the theoretical foundations in Section III-A, we develop a solution framework for the admissibility problem.

Observe that, through Definitions 1 and $2, \mathcal{S}^{\text {uncertain }}$ is admissible for $\mathbf{v}^{\text {initial }}$ if there exists a set $\mathcal{V}$ such that

(O1) $\mathcal{V} \subseteq \mathcal{V}^{\text {secure }}$

(O2) $\mathbf{v}^{\text {initial }} \in \mathcal{V}$;

(O3) $\mathcal{S}^{\text {uncertain }}$ is a domain of $\mathcal{V}$-control.

By this observation, our framework consists in constructing a set $\mathcal{V}$, as large as possible, such that $(\mathrm{O} 1)-(\mathrm{O} 3)$ are satisfied. In the first step, we find a large open set $\tilde{\mathcal{V}}$ such that its closure $\operatorname{cl}(\tilde{\mathcal{V}})$ is non-singular and convex, using some explicit conditions on non-singularity (e.g., the ones given in [1], [18], [19]). Then, let $\mathcal{V}$ be the intersection of $\tilde{\mathcal{V}}$ and $\mathcal{V}^{\text {secure }}$, which fulfills (O1). Note, the obtained $\mathcal{V}$ is open and non-singular. Moreover, by Theorem 2, it is also a domain of uniqueness.

In the second step, we first verify $(\mathrm{O} 2)$ by inspection. Then, for (O3), we test whether $\forall \mathbf{s} \in \mathcal{S}^{\text {uncertain }}$, there is no corresponding state at the boundary $\partial \mathcal{V}$. This is done by solving a number of feasibility problems (see Step 2 below). By Theorem 3, this will guarantee that there exists a corresponding state $\mathbf{v} \in \mathcal{V}$ for every $\mathbf{s} \in \mathcal{S}^{\text {uncertain }}$. Further, by Theorem 1, this will guarantee that $(\mathrm{O} 3)$ is satisfied.

Specifically, the framework is described below.
Framework Given an initial state $\mathbf{v}^{\text {initial }}$ and a connected uncertainty set $\mathcal{S}^{\text {uncertain }}$ such that $\mathbf{v}^{\text {initial }} \in \mathcal{V}^{\text {secure }}$ and $\mathbf{F}\left(\mathbf{v}^{\text {initial }}\right) \in \mathcal{S}^{\text {uncertain }}$ (i.e., (I1)-(I2) are satisfied).

(Step 1) Construct $\mathcal{V}$ as follows:

- Find some appropriate continuous functions $f_{\ell}$, $\ell \in\left\{L^{\text {secure }}+1, \ldots, L\right\}$ such that $\tilde{\mathcal{V}}=\left\{\mathbf{v}: f_{\ell}(\mathbf{v})>0, \ell=L^{\text {secure }}+1, \ldots, L\right\}$ is large and its closure is non-singular and convex; ${ }^{3}$

- Then, let $\mathcal{V}=\left\{\mathbf{v}: f_{\ell}(\mathbf{v})>0, \ell=1, \ldots, L\right\}$ (i.e., let $\mathcal{V}=\tilde{\mathcal{V}} \cap \mathcal{V}^{\text {secure }}$ ).

(Step 2) Test whether

- $\mathbf{v}^{\text {initial }} \in \mathcal{V}$;

- The following problems are infeasible for all $\ell$.

$[\mathbf{P O}(\ell)] \quad$ Find $\mathbf{v}$ such that :

$$
\begin{aligned}
& f_{\ell^{\prime}}(\mathbf{v}) \geq 0, \forall \ell^{\prime} \in\{1, \ldots, L\} \backslash\{\ell\}, \\
& f_{\ell}(\mathbf{v})=0, \\
& \mathbf{F}(\mathbf{v}) \in \mathcal{S}^{\text {uncertain }} .
\end{aligned}
$$

If both tests succeed, then declare that $\mathcal{S}^{\text {uncertain }}$ is admissible for $\mathbf{v}^{\text {initial }}$. Otherwise, we are unsure of the admissibility.

Remark 1. In the related work [7], a convex set $\mathcal{V}$ is constructed such that (i) it satisfies a special form of inequalities $f_{\ell}(\mathbf{v})>0$, and (ii) for any $\mathbf{s}$ in an analytically specified neighborhood of $\mathbf{s}^{\text {initial }}$, there exists at least one corresponding state in $\mathcal{V}$. Compared to the $\mathcal{V}$ obtained by our framework, the one obtained in [7] cannot handle general forms of $f_{\ell}(\mathbf{v})>0$ and might be neither non-singular nor a domain of uniqueness.

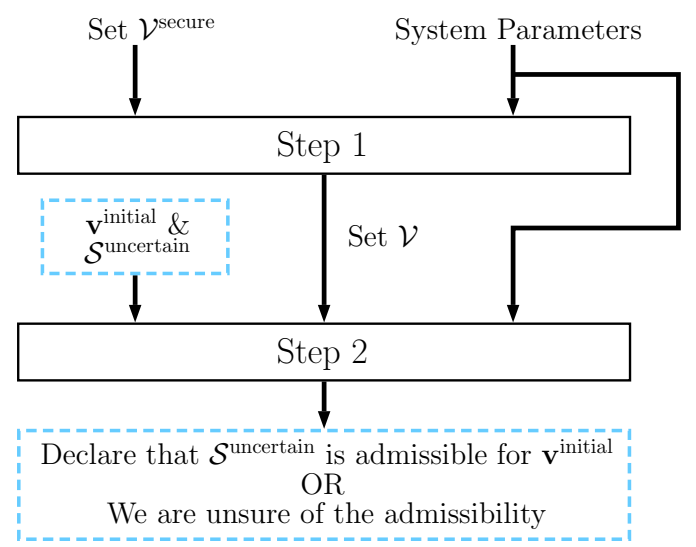

Fig. 3. Flow chart of the framework.

For the proposed framework, we highlight its structure in Figure 3 and propose the following theorem on its validity. Note that the theorem is formulated from a theoretical perspective and does not account for the finite numerical precision in practical implementation.

Theorem 4. The above framework is correct in the sense that, whenever it declares $\mathcal{S}^{\text {uncertain }}$ admissible for $\mathbf{v}^{\text {initial }}$, it is so.

Proof. We need to show that $\mathcal{S}^{\text {uncertain }}$ is a domain of $\mathcal{V}$ control. By Theorem 1, as $\mathcal{V}$ is already open and non-singular,

\footnotetext{
${ }^{3}$ Note that $\operatorname{cl}(\tilde{\mathcal{V}})=\left\{\mathbf{v}: f_{\ell}(\mathbf{v}) \geq 0, \ell=L^{\text {secure }}+1, \ldots, L\right\}$.
} 
we only need to prove that $\forall \mathbf{s} \in \mathcal{S}^{\text {uncertain }}$, there is a unique $\mathbf{v} \in \mathcal{V}$ such that $\mathbf{F}(\mathbf{v})=\mathbf{s}$.

According to Theorem $2, \mathcal{V}$ is a domain of uniqueness, because it is included in a non-singular and convex set. In this way, it suffices to show that, for any $\mathbf{s} \in \mathcal{S}^{\text {uncertain }}$, there exists a $\mathbf{v} \in \mathcal{V}$ such that $\mathbf{F}(\mathbf{v})=\mathbf{s}$. For this purpose, we check the four conditions in Theorem 3 .

In Theorem 3, the 1st and the 2nd conditions are automatically satisfied due to Assumption 1. The 3rd condition follows from $\mathbf{F}\left(\mathbf{v}^{\text {initial }}\right) \in \mathcal{S}^{\text {uncertain }}$.

Now, let us focus on the 4 th condition. Since $\mathrm{P} 0(\ell)$ is infeasible for all $\ell$, we have that the set

$$
\mathbf{F}\left(\bigcup_{\ell=1}^{L}\left\{\mathbf{v}: f_{\ell}(\mathbf{v})=0 \text { and } f_{\ell^{\prime}}(\mathbf{v}) \geq 0, \ell^{\prime} \in\{1, \ldots, L\} \backslash\{\ell\}\right\}\right)
$$

has an empty intersection with $\mathcal{S}^{\text {uncertain }}$. Therefore, we can complete the proof by showing that the boundary $\partial \mathcal{V}$ is contained in the set

$$
\bigcup_{\ell=1}^{L}\left\{\mathbf{v}: f_{\ell}(\mathbf{v})=0 \text { and } f_{\ell^{\prime}}(\mathbf{v}) \geq 0, \ell^{\prime} \in\{1, \ldots, L\} \backslash\{\ell\}\right\} .
$$

Consider that all $f_{\ell}$ are continuous and the topological boundary of $\mathcal{V}$ is the set of points that are both limit points of $\mathcal{V}$ and limit points of the complement of $\mathcal{V}$. If $\mathbf{v} \in \partial \mathcal{V}$, then $\mathbf{v}$ is the limit of some infinite sequence $\mathbf{v}^{(n)} \in \mathcal{V}$, thus $f_{\ell}\left(\mathbf{v}^{(n)}\right)>0$ and $f_{\ell}(\mathbf{v}) \geq 0$ for all $\ell$. Also, $\mathbf{v}$ is the limit of some infinite sequence $\mathbf{v}^{\prime(n)}$ outside $\mathcal{V}$. Since there are only finitely many inequalities, there must be at least one inequality, say with index $\ell^{\star}$, such that $f_{\ell^{\star}}\left(\mathbf{v}^{\prime(n)}\right) \leq 0$ for an infinite number of indexes $n$. It follows that $f_{\ell^{\star}}(\mathbf{v}) \leq 0$ and thus $f_{\ell^{\star}}(\mathbf{v})=0$.

Observe that, in Step 1 of the framework, we attempt to find a set $\mathcal{V}$ that is as large as possible. This contrasts with what happened when we applied the theory of $\mathcal{V}$-control in Section II-D; there, $\mathcal{V}$ should be large enough, but not too large. Indeed, the fact that $\mathcal{S}^{\text {uncertain }}$ is a domain of $\mathcal{V}$-control might not automatically extend to a superset of $\mathcal{V}$. On the contrary, when using Theorems 2 and 3 as we do here, it is always beneficial to have $\mathcal{V}$ as large as possible. This follows from the theorem given below.

Theorem 5. Assume that we are given two implementations, $\mathbf{A}$ and $\mathbf{A}^{\prime}$, of the framework, where, in Step 1, $\mathbf{A}$ constructs $\mathcal{V}$ and $\mathbf{A}^{\prime}$ constructs $\mathcal{V}^{\prime} \supseteq \mathcal{V}$. Also assume that the ground truth is that $\mathcal{S}^{\text {uncertain }}$ is admissible for $\mathbf{v}^{\text {initial }}$. If $\mathbf{A}$ declares $\mathcal{S}^{\text {uncertain }}$ admissible for $\mathbf{v}^{\text {initial }}$, then so does $\mathbf{A}^{\prime}$.

Proof. By hypothesis, we have that $\mathcal{S}^{\text {uncertain }}$ and $\mathcal{V}$ satisfy the four conditions in Theorem 3. In addition, in Step 1 of $\mathbf{A}^{\prime}$, we have an open set $\tilde{\mathcal{V}}^{\prime}$ such that (i) its closure $\operatorname{cl}\left(\tilde{\mathcal{V}}^{\prime}\right)$ is nonsingular and convex, and (ii) $\mathcal{V}^{\prime}=\tilde{\mathcal{V}}^{\prime} \cap \mathcal{V}^{\text {secure }}$. All we need to show is that $\mathcal{S}^{\text {uncertain }}$ and $\mathcal{V}^{\prime}$ satisfy the four conditions in Theorem 3, which we do next.

First, the open set $\tilde{\mathcal{V}}^{\prime}$ is non-singular, since its closure $\operatorname{cl}\left(\tilde{\mathcal{V}}^{\prime}\right)$ is non-singular. Therefore, by Assumption $1, \mathcal{V}^{\prime}=\tilde{\mathcal{V}}^{\prime} \cap \mathcal{V}^{\text {secure }}$ is bounded, open and non-singular, which means that condition 1) in Theorem 3 holds.

Next, condition 2) in Theorem 3 holds by hypothesis, and condition 3 ) in Theorem 3 holds because (i) $\mathbf{F}(\mathcal{V}) \cap \mathcal{S}$ is nonempty; (ii) $(\mathbf{F}(\mathcal{V}) \cap \mathcal{S}) \subseteq\left(\mathbf{F}\left(\mathcal{V}^{\prime}\right) \cap \mathcal{S}\right)$.
Then, we show condition 4) in Theorem 3 by contradiction. Suppose that there exists $\mathbf{v} \in \partial \mathcal{V}^{\prime}$ such that $\mathbf{F}(\mathbf{v}) \in \mathcal{S}^{\text {uncertain }}$. Let $\mathbf{F}(\mathbf{v})=\mathbf{s}$ and consider: (i) $\mathcal{S}^{\text {uncertain }}$ and $\mathcal{V}$ satisfy all the conditions in Theorem 3; (ii) $\mathcal{V} \subseteq \mathcal{V}^{\prime}$, both of which are open and have empty intersection with $\partial \mathcal{V}^{\prime}$. We have that there exists $\mathbf{v}^{*} \in \mathcal{V}$ such that $\mathbf{F}\left(\mathbf{v}^{*}\right)=\mathbf{s}$ and $\mathbf{v}^{*} \neq \mathbf{v}$. In this way, we obtain two distinct elements in $\operatorname{cl}\left(\tilde{\mathcal{V}}^{\prime}\right)$ that both correspond to $\mathbf{s} \in \mathcal{S}^{\text {uncertain }}$. However, this creates a contradiction, as $\operatorname{cl}\left(\tilde{\mathcal{V}}^{\prime}\right)$ is a domain of uniqueness according to Theorem 2. As a result, we have that condition 4 ) in Theorem 3 holds.

\section{Application to AC Electrical Networks: SOLUTION}

In this section, we apply the proposed framework in Section III-B to solve the admissibility problem in AC electrical networks that is formulated in Section II-B. Specifically, we develop a method in Sections IV-A and IV-B, which uses the framework and correspondingly has two steps. Next, in Section IV-C, we describe the computational complexity and the implementation issues of this method.

\section{A. Step 1 of the Method}

As in the first step of our framework, we need to find an open set $\tilde{\mathcal{V}}$ such that its closure $\operatorname{cl}(\tilde{\mathcal{V}})$ is non-singular and convex. First, take into account the following (Chap. 5 of [20]):

- The singularity of Jacobian matrix $\mathbf{J}_{\mathbf{F}}$ usually occurs due to high power generation and consumption;

- High power generation and consumption are linked to large magnitudes of the branch and nodal currents.

Therefore, for $\operatorname{cl}(\tilde{\mathcal{V}})$ to be non-singular, we need to ensure that no state in $\operatorname{cl}(\tilde{\mathcal{V}})$ has very large branch and nodal currents. Based on this consideration, we let $\tilde{\mathcal{V}}$ be

$$
\begin{aligned}
\tilde{\mathcal{V}}=\{\mathbf{v}: & \tilde{f}_{j k}^{\mathrm{I}, \text { branch }}(\mathbf{v})>0, \forall j k \in \mathcal{E}, \\
& \left.\tilde{f}_{j}^{\mathrm{I}, \text { node }}(\mathbf{v})>0, \forall j \in \mathcal{N}^{P Q}\right\}
\end{aligned}
$$

with

$$
\begin{gathered}
\tilde{f}_{j k}^{\mathrm{I}, \text { branch }}(\mathbf{v})=-\left|a_{j k} v_{0}+\mathbf{c}_{j k}^{T} \mathbf{v}\right|^{2}+\left(I_{j k}^{\text {branch }}\right)^{2}, \\
\tilde{f}_{j}^{\mathrm{I}, \text { node }}(\mathbf{v})=-\left|\operatorname{Row}_{j}\left(\mathbf{Y}_{L L}\right)(\mathbf{v}-\mathbf{w})\right|^{2}+\left(I_{j}^{\text {node }}\right)^{2} .
\end{gathered}
$$

Here, $\operatorname{Row}_{j}()$ means the $j$-th row of a matrix, and $I_{j k}^{\text {branch }}$, $I_{j}^{\text {node }}$ are some positive auxiliary constants. As can be seen,

- The set $\tilde{\mathcal{V}}$ defined in (8) is open and its closure is convex;

- (9)-(10) together specify the functions $f_{\ell}(\mathbf{v}), \ell \in$ $\left\{L^{\text {secure }}+1, \ldots, L\right\}$ in Step 1 of the framework.

In what follows, our task is to find appropriate values for constants $I_{j k}^{\text {branch }}, j k \in \mathcal{E}$ and $I_{j}^{\text {node }}, j \in \mathcal{N}^{P Q}$ such that the set $\operatorname{cl}(\tilde{\mathcal{V}})$ is non-singular. To this end, recall that a necessary condition for $\mathbf{J}_{\mathbf{F}}(\mathbf{v})$ to be singular is given by (8) in [19] as:

$$
\exists m \in \mathcal{N}^{P Q} \text { such that } \sum_{n=1}^{N}\left|\left(\mathbf{Y}_{L L}^{-1}\right)_{m, n} i_{n}\right| \geq\left|v_{m}\right|,
$$

where $(\cdot)_{m, n}$ is the entry of the $m$-th row, $n$-th column in a matrix. By contraposition, a sufficient condition on the nonsingularity of $\mathbf{J}_{\mathbf{F}}(\mathbf{v})$ is obtained as follows:

$$
\sum_{n=1}^{N}\left|\left(\mathbf{Y}_{L L}^{-1}\right)_{m, n} i_{n}\right|<\left|v_{m}\right|, \forall m \in \mathcal{N}^{P Q} \text {. }
$$


If this sufficient condition is satisfied $\forall \mathbf{v} \in \operatorname{cl}(\tilde{\mathcal{V}})$, then $\operatorname{cl}(\tilde{\mathcal{V}})$ is non-singular. Thus, we have the following proposition, where $\|\cdot\|_{1}$ is the $\ell_{1}$ norm. The corresponding proof can be found in Appendix-B.

Proposition 1. The closure of the set $\tilde{\mathcal{V}}$ defined in (8) is nonsingular if the following problems are infeasible for all $m, n \in$ $\mathcal{N}^{P Q}$ and $\psi, \phi \in\{1,-1\}$.

$[\mathbf{P 1}(m, n, \psi, \phi)]$ Find $\mathbf{v}$ such that :

$$
\begin{aligned}
& \tilde{f}_{j k}^{\mathrm{I}, \text { branch }}(\mathbf{v}) \geq 0, \forall j k \in \mathcal{E}, \\
& \tilde{f}_{j}^{\mathrm{I}, \operatorname{node}^{\prime}}(\mathbf{v}) \geq 0, \forall j \in \mathcal{N}^{P Q}, \\
& \left\|\operatorname{Row}_{m}\left(\mathbf{Y}_{L L}^{-1}\right)\right\|_{1}\left(\psi \operatorname{Re}\left(\operatorname{Row}_{n}\left(\mathbf{Y}_{L L}\right)(\mathbf{v}-\mathbf{w})\right)\right. \\
& \left.+\phi \operatorname{Im}\left(\operatorname{Row}_{n}\left(\mathbf{Y}_{L L}\right)(\mathbf{v}-\mathbf{w})\right)\right) \geq\left|v_{m}\right|, \\
& \psi \operatorname{Re}\left(\operatorname{Row}_{n}\left(\mathbf{Y}_{L L}\right)(\mathbf{v}-\mathbf{w})\right) \geq 0 \\
& \phi \operatorname{Im}\left(\operatorname{Row}_{n}\left(\mathbf{Y}_{L L}\right)(\mathbf{v}-\mathbf{w})\right) \geq 0 .
\end{aligned}
$$

By above reasoning, we develop the first step of the method as follows. Specifically, we fix some large values for $I_{j k}^{\text {branch }}, \forall j k \in \mathcal{E}$ and attempt to find as large as possible $I_{j}^{\text {node }}, \forall j \in \mathcal{N}^{P Q}$ such that $\mathrm{P} 1(m, n, \psi, \phi)$ are all infeasible.

\section{Method (Step 1)}

(1-a) First, fix $I_{j k}^{\text {branch }}=I_{j k}^{\max }, \forall j k \in \mathcal{E} .^{4}$

(1-b) Then, choose some positive reference values for $I_{j}^{\text {node }}$ and denote them by $\hat{I}_{j}^{\text {node }}, j \in \mathcal{N}^{P Q}$. We could let $\hat{I}_{j}^{\text {node }}$ be the peak nodal current magnitude at bus $j$ in real-world operation, or just let all $\hat{I}_{j}^{\text {node }}$ be the same.

(1-c) Next, let $I_{j}^{\text {node }}=\lambda \hat{I}_{j}^{\text {node }}, \forall j \in \mathcal{N}^{P Q}$, where $\lambda$ is a positive scaling factor that will vary in the subsequent step. In addition, for every $j$, specify some positive threshold $I_{j}^{\text {threshold }}$, which should be an upper bound on the largest possible nodal current magnitude at bus $j$. These thresholds are used as stopping criteria of the subsequent step.

(1-d) We start with a sufficiently small $\lambda \in$ $\left(0, \min _{j} I_{j}^{\text {threshold }} / \hat{I}_{j}^{\text {node }}\right) \quad$ (such that the problems $\mathrm{P} 1(m, n, \psi, \phi)$ are all infeasible) and gradually increase it by a fixed step size $\Delta \lambda$, until (i) $\mathrm{P} 1(m, n, \psi, \phi)$ is no longer simultaneously infeasible for all $m, n \in \mathcal{N}^{P Q}$, $\psi, \phi \in\{1,-1\}$; or (ii) $\lambda \geq \min _{j} I_{j}^{\text {threshold }} / \hat{I}_{j}^{\text {node }}$.

(1-e) With the penultimate value of $\lambda$, we obtain $I_{j}^{\text {node }}, \forall j \in$ $\mathcal{N}^{P Q}$ and the set $\tilde{\mathcal{V}}$ that is defined in (8).

(1-f) Last, we let $\mathcal{V}=\tilde{\mathcal{V}} \cap \mathcal{V}^{\text {secure }}$.

\section{B. Step 2 of the Method}

According to the second step of the proposed framework, our main task amounts to checking the infeasibility of $\mathrm{P} 0(\ell)$ for every $\ell \in\{1, \ldots, L\}$ as explained in Section III-B.

Observe that, for each problem $\mathrm{P} 0(\ell)$, we have

- $f_{\ell}(\mathbf{v}), \ell \in\{1, \ldots, L\}$ are all polynomial in $\operatorname{Re}(\mathbf{v}), \operatorname{Im}(\mathbf{v})$;

- $\mathbf{F}(\mathbf{v})$ is a system of polynomials in $\operatorname{Re}(\mathbf{v})$ and $\operatorname{Im}(\mathbf{v})$.

Therefore, the constraints in $\mathrm{P} 0(\ell)$ are all polynomial equalities / inequalities if we add the following assumption.

\footnotetext{
${ }^{4} \mathrm{We}$ choose these values because they reduce the complexity in the second step of our method. For details, see Sections IV-B and IV-C.
}

Assumption 2. $\mathcal{S}^{\text {uncertain }}$ is the Cartesian product of $\mathcal{S}_{j}^{\text {uncertain }}, \forall j \in \mathcal{N}^{P Q}$, and each $\mathcal{S}_{j}^{\text {uncertain }}$ is either a convex polygon or a singleton.

Indeed, under this assumption, the constraints $\mathbf{F}(\mathbf{v}) \in$ $\mathcal{S}^{\text {uncertain }}$ can be equivalently written as a collection of inequalities that are polynomial in $\operatorname{Re}(\mathbf{v})$ and $\operatorname{Im}(\mathbf{v})$, with the following form:

$$
\mu_{l}^{\mathrm{re}} \operatorname{Re}\left((\mathbf{F}(\mathbf{v}))_{l}\right)+\mu_{l}^{\mathrm{im}} \operatorname{Im}\left((\mathbf{F}(\mathbf{v}))_{l}\right)+\mu_{l}^{\text {const }} \geq 0,
$$

where $\mu_{l}^{\mathrm{re}}, \mu_{l}^{\mathrm{im}}, \mu_{l}^{\text {const }} \in \mathbb{R}$.

Note that the polynomial problems $\mathrm{P} 0(\ell)$ are not convex. For this reason, we could apply convex relaxation to them and check whether the relaxed problems are infeasible. Indeed, the infeasibility of the relaxed problem implies the infeasibility of the original problem. As proposed in [21], these nonconvex polynomial problems can be effectively approximated by a hierarchy of semi-definite relaxations. This hierarchy is arranged by a positive integer called relaxation order. As the relaxation order increases, the relaxed problem becomes closer to the original problem, in terms of the feasibility. Despite the theoretical beauty of this hierarchy of relaxations, as the number of variables and the relaxation order increase, it gradually becomes computationally intractable. To cope with this issue, a sparsity-exploiting counterpart of this hierarchy is developed later in [22], [23], where the level of sparsity depends mainly on the cross terms in the polynomial constraints. In [24]-[26], very nice examples can be found concerning the application of these hierarchies to electrical networks.

Taking the above into consideration, we develop the second step of the method below.

\section{Method (Step 2)}

(2-a) Given the set $\mathcal{V}$ obtained in Method (Step 1), check whether $\mathbf{v}^{\text {initial }} \in \mathcal{V}$.

(2-b) With the same $\mathcal{V}$ and the sparsity-exploiting hierarchy of semi-definite relaxations in [22], [23], check whether the relaxed $\mathrm{P} 0(\ell)$ are all infeasible for some relaxation order. (Note, under Assumption 2, an empirically good choice of the relaxation order is 2.)

(2-c) If both (2-a) and (2-b) are true, then we declare that $\mathcal{S}^{\text {uncertain }}$ is admissible for $\mathbf{v}^{\text {initial }}$. Otherwise, we are unsure of the admissibility.

Remark 2. For the sparsity-exploiting hierarchy of semidefinite relaxations, a concise tutorial can be found in [27] and a thorough description can be found in [28].

\section{Computational Complexity and Implementation Issues}

We give below a proposition on the computational complexity of the method. It is proved in Appendix-C and uses the following notations $(\operatorname{card}(\cdot)$ means cardinality):

- $\mathcal{C}_{r}, r=1, \ldots, R$ are the maximal cliques of a chordal extension [29] of the graph $\left(\mathcal{N}^{\text {poly }}, \mathcal{E}^{\text {poly }}\right)$, where $R$ is the total number of maximal cliques and

$-\mathcal{N}^{\text {poly }}=\{1, \ldots, 2 N\}$, 
- $\mathcal{E}^{\text {poly }}$ collects $\{j, h\} \subseteq \mathcal{N}^{\text {poly }}$ such that the $j$-th and $h$-th entries of vector $\left(\operatorname{Re}(\mathbf{v})^{T}, \operatorname{Im}(\mathbf{v})^{T}\right)^{T}$ are coupled by at least one polynomial constraint in $\mathrm{P} 0$,

- $\mathcal{K}=\{1, \ldots, 3 N+\operatorname{card}(\mathcal{E})+\Upsilon\}$, where $\Upsilon$ represents the number of polynomial inequalities in $\mathbf{F}(\mathbf{v}) \in \mathcal{S}^{\text {uncertain }}$,

- $\theta(k)$ is an index mapping from $\mathcal{K}$ to $\{1, \ldots, R\}$ such that $\mathcal{I}_{k} \subseteq \mathcal{C}_{\theta(k)}$, where $\mathcal{I}_{k}$ collects $j \in \mathcal{N}^{\text {poly }}$ such that the $j$-th entry of vector $\left(\operatorname{Re}(\mathbf{v})^{T}, \operatorname{Im}(\mathbf{v})^{T}\right)^{T}$ explicitly shows up in the $k$-th polynomial constraint of $\mathrm{P} 0$,

- $\omega$ is the relaxation order and $\mathcal{A}_{\omega}^{\mathcal{C}_{r}}=\left\{\boldsymbol{\alpha} \in \mathbb{N}^{2 N}: \alpha_{j}=\right.$ $\left.0, \forall j \notin \mathcal{C}_{r}, \quad \sum_{j=1}^{2 N} \alpha_{j} \leq \omega\right\}$.

Proposition 2. For the proposed method in Sections IV-A and $I V-B$, we have:

- The complexity of Step 1 is equal to that of at most $4 N^{2}\left\lceil\min _{j} I_{j}^{\text {threshold }} /\left(\hat{I}_{j}^{\text {node }} \Delta \lambda\right)\right\rceil$ second-order cone feasibility problems, where $\lceil\cdot\rceil$ means ceiling. Each feasibility problem has $2 N$ real variables and $3+N+\operatorname{card}(\mathcal{E})$ inequality constraints.

- Under Assumption 2, the complexity of Step 2 is equal to that of $3 N+\operatorname{card}(\mathcal{E})$ semi-definite feasibility problems. Each feasibility problem has $\operatorname{card}\left(\bigcup_{r=1}^{R} \mathcal{A}_{2 \omega}^{\mathcal{C}_{r}}\right)$ real variables and an LMI constraint with matrices of size $\sum_{k=1}^{\operatorname{card}(\mathcal{K})} \operatorname{card}\left(\mathcal{A}_{\omega-1}^{\mathcal{C}_{\theta(k)}}\right)+\sum_{r=1}^{R} \operatorname{card}\left(\mathcal{A}_{\omega}^{\mathcal{C}_{r}}\right)$.

Furthermore, we present some implementation issues as follows, which are useful for online applications.

1) For a given network configuration (i.e., topology, line parameters, etc.), the first step of the proposed method needs to be implemented only once.

2) In the first step of the proposed method, the infeasibility of each $\mathrm{P} 1(m, n, \psi, \phi)$ can be checked independently. Thus, the first step of the method can be implemented in parallel through a multi-core CPU/GPU or a networked computing infrastructure; this is of significance for relatively large $N$. Similarly, in the second step of our method, the infeasibility of each relaxed $\mathrm{P} 0(\ell)$ can also be checked independently. Therefore, the second step of the method can be implemented in parallel as well.

From the theoretical perspective, the complexity of a secondorder cone feasibility problem and the complexity of a semidefinite feasibility problem remain as open research topics [30]-[32]. Despite this fact, in the numerical evaluations of the following section, the corresponding feasibility problems take at most around a minute cumulatively for both steps of the proposed method, as it is shown in Table I.

\section{Numerical ExAMPLES IN AC EleCtricAL NETWORKS}

In this section, we evaluate the performance of the proposed method in Sections IV-A and IV-B via two examples. The first example has a size that is typical for practical applications in electrical microgrids; the second example has a size that is typical for practical applications in electrical distribution networks. In both examples, we assume that (i) the slackbus voltage is 1 p.u.; (ii) the relaxation order in the second step of our method is 2 . Here, we note that the results are generated using 3.0 GHz Intel Core i7 CPU and $32 \mathrm{~GB} 1867$
MHz memory. In particular, we implement the method using MATLAB tools YALMIP, Mosek and SparsePOP.

\section{A. Example 1}

We consider the residential part of the CIGRE North American LV Distribution Network [33] and make it meshed by adding several transmission lines. In Figure 4, we show the topology of the modified network, where (i) the black lines represent the transmission lines in the unmodified network; (ii) the blue lines represent the added transmission lines.

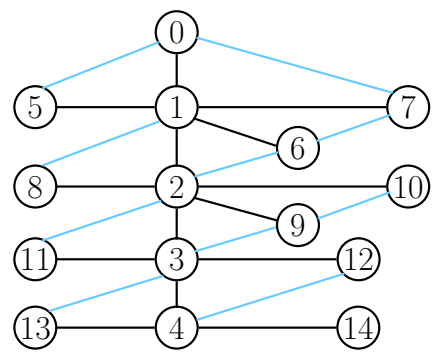

Fig. 4. Topology of the modified CIGRE North American LV Distribution Network (residential part).

The impedances of the added transmission lines are the same as that of the transmission line between buses 2 and 9 .

For this network, we assume that each of the buses 1-4 has an extra energy source. Moreover,

- Each of these sources is balanced across the neutral line;

- Each of these sources has a real-valued power in [20(1$\kappa), 20(1+\kappa)] \mathrm{kW}$, where scalar $\kappa \in[0,1)$;

- These sources are independent of each other.

By fixing the peak powers given in [33] for the other buses, we construct a set $\mathcal{S}^{\text {uncertain }}$. Now, let (i) $\mathrm{s}^{\text {initial }}$ be the central point in $\mathcal{S}^{\text {uncertain }}$, and (ii) $\mathbf{v}^{\text {initial }}$ be its corresponding state that is guaranteed to be unique around $\mathrm{w}$ by conditions in [3]. To ensure that $\mathbf{v}^{\text {initial }}$ satisfies the security constraints, let

$$
\begin{aligned}
& \text { - } V_{j}^{\min }=0.95 \text { p.u. } \forall j \in \mathcal{N}^{P Q} \text {; } \\
& \text { - } V_{j}^{\max }=1.05 \text { p.u. } \forall j \in \mathcal{N}^{P Q} \text {; } \\
& \text { - } I_{j k}^{\max }=0.4 \text { p.u. } \forall j k \in \mathcal{E} .
\end{aligned}
$$

Our goal is to find the maximum value of $\kappa$ such that $\mathcal{S}^{\text {uncertain }}$ is admissible for $\mathbf{v}^{\text {initial }}$.

In the first step of our method, we take $I_{j k}^{\text {branch }}=I_{j k}^{\max }, j k \in$ $\mathcal{E}$. With these $I_{j k}^{\text {branch }}, j k \in \mathcal{E}$, one valid choice of $I_{j}^{\text {node }}, j \in$ $\mathcal{N}^{P Q}$ is: $I_{j}^{\text {node }}=0.7$ p.u. $\forall j \in \mathcal{N}^{P Q}$. In this way, we have obtained a set $\mathcal{V}$. To show that $\mathcal{V}$ captures a non-trivial portion of $\mathcal{V}^{\text {secure }}$, we provide a visualization in Figure 5. Each plot in this figure contains a cross section of $\mathcal{V}$ and a cross section of $\mathcal{V}^{\text {secure }}$; they are drawn by varying one complex nodal voltage (at bus 1 or 14) and fixing all the other complex nodal voltages to their initial values. In each plot, the cross section of $\mathcal{V}$ is represented by filled squares, and the cross section of $\mathcal{V}^{\text {secure }}$ is represented by all squares (filled and unfilled). As can be seen, (i) in the top plot, the cross section of $\mathcal{V}$ captures a large portion of its counterpart of $\mathcal{V}^{\text {secure}}$; (ii) in the bottom plot, the cross section of $\mathcal{V}$ is the same as its counterpart of $\mathcal{V}^{\text {secure }}$. Next, using the second step of our method, we find that $\mathbf{v}^{\text {initial }} \in \mathcal{V}$ and the maximum value for $\kappa$ 

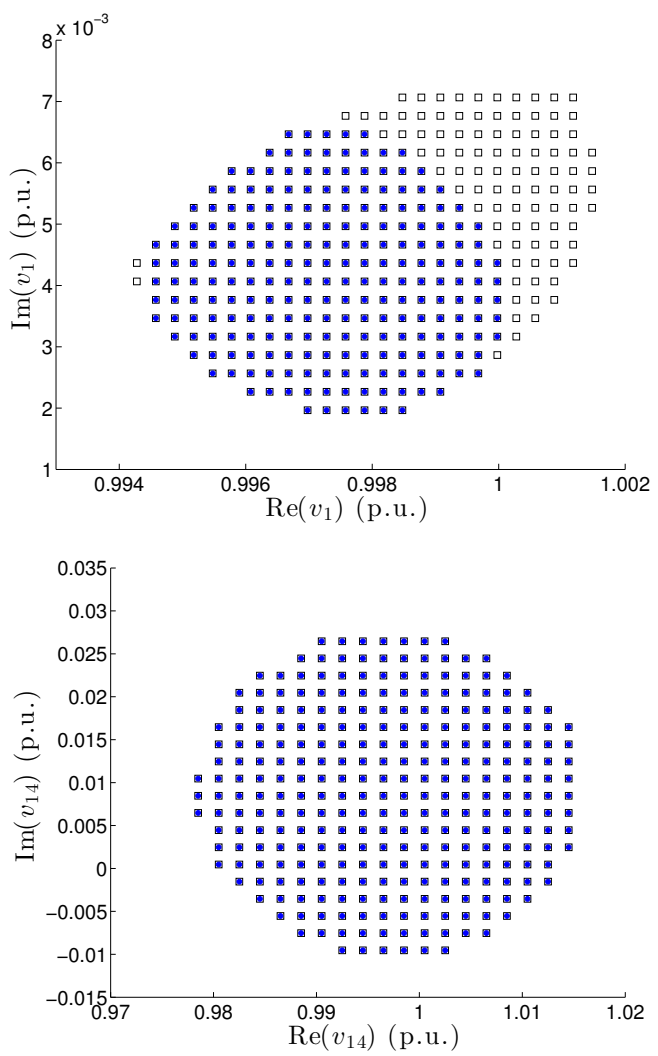

Fig. 5. (Top) Cross sections of $\mathcal{V}$ and $\mathcal{V}^{\text {secure }}$, obtained by varying $v_{1}$ and fixing $v_{j}=v_{j}^{\text {initial }}, j \neq 1$. (Bottom) Cross sections of $\mathcal{V}$ and $\mathcal{V}^{\text {secure }}$, obtained by varying $v_{14}$ and fixing $v_{j}=v_{j}^{\text {initial }}, j \neq 14$. In each plot, the cross section of $\mathcal{V}$ is represented by filled squares, and the cross section of $\mathcal{V}^{\text {secure }}$ is represented by all squares (filled and unfilled).

to preserve admissibility is 0.48 (i.e., solvers declare numerical infeasibility for all relaxed $\mathrm{P} 0(\ell)$ when $\kappa<0.48)$.

In this example, the measured execution time of parallel implementation is reported in Table I. Apart from this, for each tested $\kappa$ that does not preserve admissibility, the DIMACS errors [34] are typically between $10^{-7}$ and $10^{-4}$, which means an acceptable level of numerical accuracy.

For evaluating the tightness of the method, we apply grid searching to numerically compute the true maximum value of $\kappa$ that preserves admissibility. Specifically, we first discretize the set $\mathcal{S}^{\text {uncertain }}$ with an appropriate resolution (e.g., each of the 4 real-valued uncertain dimensions divided into 25 intervals), which gives us a large number of candidate values for the uncertain injection. Then for each candidate value of the injection, we compute the corresponding state that is guaranteed to be unique around $\mathbf{v}^{\text {initial }}$ by conditions in [3]. Finally, we check whether there exists a computed state that does not belong to $\mathcal{V}^{\text {secure }}$. Via this grid searching, we obtain that the true maximum value for $\kappa$ to preserve admissibility is 0.52 , which is close to 0.48 and means that our proposed method is tight. It should be noticed that the grid searching is computationally much heavier than the proposed method.
TABLE I

MeAsured TIME FOR EXAMPLES 1 AND 2 (IN SECONDS)

\begin{tabular}{lcc}
\hline & Step 1 & Step 2 \\
\hline Example 1 & $1-2$ & $11-19$ \\
Example 2 & $4-5$ & $49-57$ \\
\hline
\end{tabular}

\section{B. Example 2}

We consider the IEEE 34-Bus Test Feeder [35] and make it balanced by taking line type 602 in [35] for all transmission lines. We assume that each of the buses $812,850,852$ has a device that can both generate and consume power. Moreover,

- Each of these devices is balanced across the phases;

- Each of these devices has a complex-valued power in $[-100 \kappa, 100 \kappa] \times[-60 \kappa, 60 \kappa] \mathrm{kVA}$, where scalar $\kappa \in[0, \infty)$;

- These devices are independent of each other.

By fixing the powers of the other buses to their phase-average values given in [35], we construct a set $\mathcal{S}^{\text {uncertain }}$. Again, let (i) $\mathbf{s}^{\text {initial }}$ be the central point in $\mathcal{S}^{\text {uncertain }}$, and (ii) $\mathbf{v}^{\text {initial }}$ be its corresponding state that is guaranteed to be unique around $\mathbf{w}$ by conditions in [3]. To ensure that $\mathbf{v}^{\text {initial }}$ satisfies the security constraints, let

$$
\begin{aligned}
& \text { - } V_{j}^{\min }=0.9 \text { p.u. } \forall j \in \mathcal{N}^{P Q} \text {; } \\
& \text { - } V_{j}^{\max }=1.1 \text { p.u. } \forall j \in \mathcal{N}^{P Q} \text {; } \\
& \text { - } I_{j k}^{\max }=0.4 \text { p.u. } \forall j k \in \mathcal{E} .
\end{aligned}
$$

Similarly to the previous example, we want to find the maximum value of $\kappa$ such that $\mathcal{S}^{\text {uncertain }}$ is admissible for $\mathbf{v}^{\text {initial }}$.

In the first step of our method, we take $I_{j k}^{\text {branch }}=I_{j k}^{\max }, j k \in$ $\mathcal{E}$. With these $I_{j k}^{\text {branch }}, j k \in \mathcal{E}$, one valid choice of $I_{j}^{\text {node }}, j \in$ $\mathcal{N}^{P Q}$ is: $I_{j}^{\text {node }}=0.3$ p.u. $\forall j \in \mathcal{N}^{P Q}$. In this way, we have obtained a set $\mathcal{V}$. Next, using the second step of our method, we find that $\mathbf{v}^{\text {initial }} \in \mathcal{V}$ and the maximum value for $\kappa$ to preserve admissibility is 1.42 (i.e., solvers declare numerical infeasibility for all relaxed $\mathrm{P} 0(\ell)$ when $\kappa<1.42)$.

In this example, the measured execution time of parallel implementation is reported in Table I. Besides, for each tested $\kappa$ that does not preserve admissibility, the DIMACS errors are typically between $10^{-6}$ and $10^{-4}$, which indicates an acceptable level of numerical accuracy.

For evaluating the tightness of the method, we apply again the grid searching to numerically compute the true maximum value of $\kappa$ that preserves admissibility (note that there are 6 real-valued uncertain dimensions). Via the grid searching, we obtain that the true maximum value for $\kappa$ to preserve admissibility is 1.5 , which is close to 1.42 and means that our proposed method is tight.

\section{CONCLUSIONS}

We have studied the admissibility problem in quadratic algebraic systems. In order to test the admissibility, we have established a framework, using the recently proposed $\mathcal{V}$-control. For the theoretical foundation of the framework, we show that if a set $\mathcal{V}$ of system states is non-singular and convex, then it is a domain of uniqueness. In addition, given any set $\mathcal{S}$ of system injections, we have presented topological conditions on $\mathcal{V}$ and $\mathcal{S}$ to guarantee that every injection in $\mathcal{S}$ has a corresponding state in $\mathcal{V}$. Using the framework, we have developed a concrete 
method for solving the admissibility problem in AC electrical networks, which mainly involves solving feasibility problems. The method has been then evaluated on CIGRE and IEEE networks. There exist several alternative optimization methods, such as alpha-beta theory [36], which could be used to develop other concrete methods, using the same framework.

\section{APPENDIX}

\section{A. Lemma 1 and Its Proof}

Lemma 1 (Property 1 in [37]). Given a quadratic function $\mathbf{F}(\mathbf{v})$, denote its Jacobian matrix by $\mathbf{J}_{\mathbf{F}}(\mathbf{v})$. If $\mathbf{F}(\mathbf{v})=\mathbf{F}\left(\mathbf{v}^{\prime}\right)$ and $\mathbf{v} \neq \mathbf{v}^{\prime}$, then $\mathbf{J}_{\mathbf{F}}\left(\frac{\mathbf{v}+\mathbf{v}^{\prime}}{2}\right)$ is singular.

Proof. Let $\mathbf{\Phi}(t)=\mathbf{F}\left((1-t) \mathbf{v}+t \mathbf{v}^{\prime}\right), t \in[0,1]$. Clearly, $\boldsymbol{\Phi}$ is quadratic in $t$ and can be written as $\boldsymbol{\Phi}(t)=t^{2} \mathbf{a}+t \mathbf{b}+\mathbf{c}$, where $\mathbf{a}, \mathbf{b}, \mathbf{c}$ are constant vectors. Consider that

$$
\boldsymbol{\Phi}(0)=\mathbf{F}(\mathbf{v})=\mathbf{F}\left(\mathbf{v}^{\prime}\right)=\boldsymbol{\Phi}(1),
$$

we have $\mathbf{c}=\mathbf{a}+\mathbf{b}+\mathbf{c}$. This implies that $\mathbf{a}=-\mathbf{b}$. Consequently,

$$
\begin{aligned}
& \boldsymbol{\Phi}(t)=t^{2} \mathbf{a}-t \mathbf{a}+\mathbf{c}, \\
& \frac{d \boldsymbol{\Phi}(t)}{d t}=(2 t-1) \mathbf{a} .
\end{aligned}
$$

By chain rule,

$$
\frac{d \Phi(t)}{d t}=\mathbf{J}_{\mathbf{F}}\left((1-t) \mathbf{v}+t \mathbf{v}^{\prime}\right) \cdot\left(\mathbf{v}^{\prime}-\mathbf{v}\right) .
$$

Combining (16)-(17) and taking $t=\frac{1}{2}$, we have

$$
\mathbf{J}_{\mathbf{F}}\left(\frac{\mathbf{v}+\mathbf{v}^{\prime}}{2}\right) \cdot\left(\mathbf{v}^{\prime}-\mathbf{v}\right)=\mathbf{0} \text {. }
$$

As $\mathbf{v} \neq \mathbf{v}^{\prime}$, it follows that $\mathbf{J}_{\mathbf{F}}\left(\frac{\mathbf{v}+\mathbf{v}^{\prime}}{2}\right)$ has a non-zero eigenvector corresponding to the zero eigenvalue. Thus, $\mathbf{J}_{\mathbf{F}}\left(\frac{\mathbf{v}+\mathbf{v}^{\prime}}{2}\right)$ is singular.

\section{B. Proof of Proposition 1}

Proof. First, let us construct in (19) a collection of sets:

$$
\begin{aligned}
\mathcal{V}_{m, n}= & \left\{\mathbf{v} \in \operatorname{cl}(\tilde{\mathcal{V}}):\left\|\operatorname{Row}_{m}\left(\mathbf{Y}_{L L}^{-1}\right)\right\|_{1}\left(\left|\operatorname{Re}\left(\operatorname{Row}_{n}\left(\mathbf{Y}_{L L}\right)(\mathbf{v}-\mathbf{w})\right)\right|\right.\right. \\
& \left.\left.+\left|\operatorname{Im}\left(\operatorname{Row}_{n}\left(\mathbf{Y}_{L L}\right)(\mathbf{v}-\mathbf{w})\right)\right|\right) \geq\left|v_{m}\right|\right\}
\end{aligned}
$$

where $m, n \in \mathcal{N}^{P Q}$. By inspection, we have that $\mathcal{V}_{m, n}$ is empty when $\mathrm{P} 1(m, n, \psi, \phi)$ is infeasible $\forall \psi, \phi \in\{1,-1\}$.

Next, we show that when $\mathcal{V}_{m, n}$ is empty for all $m, n \in \mathcal{N}^{P Q}$, the condition in (12) holds everywhere in $\operatorname{cl}(\tilde{\mathcal{V}})$. Specifically,

- By triangle inequality, the emptiness of $\mathcal{V}_{m, n}$ implies that the following inequality holds $\forall \mathbf{v} \in \operatorname{cl}(\tilde{\mathcal{V}})$.

$$
\left\|\operatorname{Row}_{m}\left(\mathbf{Y}_{L L}^{-1}\right)\right\|_{1}\left|\operatorname{Row}_{n}\left(\mathbf{Y}_{L L}\right)(\mathbf{v}-\mathbf{w})\right|<\left|v_{m}\right| \text {. }
$$

- Consequently for each $m \in \mathcal{N}^{P Q}$, the following inequality holds $\forall \mathbf{v} \in \operatorname{cl}(\tilde{\mathcal{V}})$, where $\|\cdot\|_{\infty}$ is the $\ell_{\infty}$ norm.

$$
\begin{aligned}
& \left\|\operatorname{Row}_{m}\left(\mathbf{Y}_{L L}^{-1}\right)\right\|_{1}\left\|\mathbf{Y}_{L L}(\mathbf{v}-\mathbf{w})\right\|_{\infty} \\
= & \left\|\operatorname{Row}_{m}\left(\mathbf{Y}_{L L}^{-1}\right)\right\|_{1}\|\mathbf{i}\|_{\infty}<\left|v_{m}\right| .
\end{aligned}
$$

- Furthermore, for each $m \in \mathcal{N}^{P Q}$, the following inequality holds $\forall \mathbf{v} \in \operatorname{cl}(\tilde{\mathcal{V}})$.

$$
\sum_{n=1}^{N}\left|\left(\mathbf{Y}_{L L}^{-1}\right)_{m, n} i_{n}\right| \leq\left\|\operatorname{Row}_{m}\left(\mathbf{Y}_{L L}^{-1}\right)\right\|_{1}\|\mathbf{i}\|_{\infty}<\left|v_{m}\right| .
$$

Thus, the closure of the set $\tilde{\mathcal{V}}$ defined in (8) is non-singular if the problems $\mathrm{P} 1(m, n, \psi, \phi)$ are all infeasible.

\section{Proof of Proposition 2}

Proof. In Step $1, I_{j k}^{\text {branch }}, \forall j k \in \mathcal{E}$ are fixed at the beginning. We have at most $\left\lceil\min _{j} I_{j}^{\text {threshold }} /\left(\hat{I}_{j}^{\text {node }} \Delta \lambda\right)\right\rceil$ choices of $\lambda$, which correspond to at $\operatorname{most}\left\lceil\min _{j} I_{j}^{\text {threshold }} /\left(\hat{I}_{j}^{\text {node }} \Delta \lambda\right)\right\rceil$ choices of $I_{j}^{\text {node }}, \forall j \in \mathcal{N}^{P Q}$. For each $\lambda$, we need to solve $4 N^{2}$ second-order cone feasibility problems. Therefore, in total, we solve at most $4 N^{2}\left\lceil\min _{j} I_{j}^{\text {threshold }} /\left(\hat{I}_{j}^{\text {node }} \Delta \lambda\right)\right\rceil$ second-order cone feasibility problems. In each of these problems, there are $2 N$ real variables and $3+N+\operatorname{card}(\mathcal{E})$ inequality constraints.

In Step 2, we have that the total number of continuous functions in equations (5)-(7) and (9)-(10) is $L=3 N+\operatorname{card}(\mathcal{E})$. This is because we fix $I_{j k}^{\text {branch }}=I_{j k}^{\max }, \forall j k \in \mathcal{E}$ in Step 1, which renders the $\operatorname{card}(\mathcal{E})$ continuous functions in (9) redundant. As a result, we have $3 N+\operatorname{card}(\mathcal{E})$ semi-definite feasibility problems to solve (namely, one feasibility problem per $\ell \in\{1, \ldots, L\}$ ). For the number of real variables and the size of matrices in the LMI constraint, explicit formulas can be found in [27].

\section{REFERENCES}

[1] C. Wang, J.-Y. Le Boudec, and M. Paolone, "Controlling the electrical state via uncertain power injections in three-phase distribution networks," IEEE Transactions on Smart Grid, 2017, DOI 10.1109/TSG.2017.2764077.

[2] A. Bernstein, L. Reyes-Chamorro, J.-Y. Le Boudec, and M. Paolone, "A composable method for real-time control of active distribution networks with explicit power setpoints. Part I: Framework," Electric Power Systems Research, vol. 125, pp. 254 - 264, 2015.

[3] C. Wang, A. Bernstein, J.-Y. Le Boudec, and M. Paolone, "Explicit conditions on existence and uniqueness of load-flow solutions in distribution networks," IEEE Transactions on Smart Grid, vol. 9, no. 2, pp. 953-962, Mar. 2018.

[4] — "Existence and uniqueness of load-flow solutions in three-phase distribution networks," IEEE Transactions on Power Systems, vol. 32, no. 4, pp. 3319-3320, Jul. 2017.

[5] M. S. Chen and W. E. Dillon, "Power system modeling," Proceedings of the IEEE, vol. 62, no. 7, pp. 901-915, Jul. 1974.

[6] J. D. Glover, M. S. Sarma, and T. Overbye, Power system analysis and design (fifth edition). Cengage Learning, 2011.

[7] D. Lee, H. D. Nguyen, K. Dvijotham, and K. Turitsyn, "Convex restriction of power flow feasible sets," IEEE Trans. on Control of Network Systems, vol. 6, no. 3, pp. 1235-1245, Sep. 2019.

[8] S. Menta, A. Hauswirth, S. Bolognani, G. Hug, and F. Dorfler, "Stability of dynamic feedback optimization with applications to power systems," 2018.

[9] T. Chen and D. Mehta, "On the network topology dependent solution count of the algebraic load flow equations," IEEE Transactions on Power Systems, vol. 33, no. 2, pp. 1451-1460, Mar. 2018.

[10] J. Marecek, T. McCoy, and M. Mevissen, "Power flow as an algebraic system," arXiv, 2016, 1412.8054v2.

[11] H. D. Nguyen and K. S. Turitsyn, "Appearance of multiple stable load flow solutions under power flow reversal conditions," in IEEE PES General Meeting, Jul. 2014.

[12] D. K. Molzahn and L. A. Roald, "Grid-aware versus grid-agnostic distribution system control: A method for certifying engineering constraint satisfaction," 2019, pp. 3445-3454.

[13] K. Dvijotham and K. Turitsyn, "Construction of power flow feasibility sets," arXiv, 2015, 1506.07191v3.

[14] S. Barratt, G. Angeris, and S. Boyd, "Automatic repair of convex optimization problems," Optimization and Engineering, vol. 22, pp. 247259, 2021.

[15] C. Gambella, J. Marecek, and M. Mevissen, "Projections onto the set of feasible inputs and the set of feasible solutions," in 2019 57th Annual Allerton Conference on Communication, Control, and Computing (Allerton). IEEE, 2019, pp. 937-943.

[16] M. Manetti, Topology. Springer, 2015.

[17] W. Rudin, Principles of mathematical analysis (3rd). McGraw, 1976.

[18] P. Kessel and H. Glavitsch, "Estimating the voltage stability of a power system," IEEE Trans. on Power Deli., vol. 1, no. 3, pp. 346-354, 1986. 
[19] Z. Wang, B. Cui, and J. Wang, "A necessary condition for power flow insolvability in power distribution systems with distributed generators," IEEE Trans. on Power Sys., vol. 32, no. 2, pp. 1440-1450, 2017.

[20] F. Milano, Power system modelling and scripting. Springer, 2010.

[21] J. B. Lasserre, "Global optimization with polynomials and the problem of moments," SIAM Journal on Opti., vol. 11, no. 3, pp. 796-817, 2001.

[22] H. Waki, S. Kim, M. Kojima, and M. Muramatsu, "Sums of squares and semidefinite program relaxations for polynomial optimization problems with structured sparsity," SIAM Journal on Optimization, vol. 17, no. 1, pp. 218-242, 2006.

[23] J. B. Lasserre, "Convergent SDP-relaxations in polynomial optimzation with sparsity," SIAM Journal on Opti., vol. 17, no. 3, pp. 822-843, 2006.

[24] C. Josz, J. Maeght, P. Panciatici, and J. C. Gilbert, "Application of the moment-SOS approach to global optimization of the opf problem," IEEE Transactions on Power Systems, vol. 30, no. 1, pp. 463-470, Jan. 2015.

[25] B. Ghaddar, J. Marecek, and M. Mevissen, "Optimal power flow as a polynomial optimization problem," IEEE Transactions on Power Systems, vol. 31, no. 1, pp. 539-546, Jan. 2016

[26] D. K. Molzahn and I. A. Hiskens, "Sparsity-exploiting moment-based relaxations of the optimal power flow problem," IEEE Transactions on Power Systems, vol. 30, no. 6, pp. 3168-3180, Nov. 2015.

[27] S. Kim and M. Kojima, "Exploiting sparsity in SDP relaxation of polynomial optimization problems," in Handbook on Semidefinite, Conic and Polynomial Optimization, M. F. Anjos and J. B. Lasserre, Eds. Published by Springer-Verlag, 2012.

[28] J. B. Lasserre, Moments, positive polynomials and their applications. Imperial College Press, 2009.

[29] J. R. S. Blair and B. W. Peyton, "An introduction to chordal graphs and clique trees," in Graph Theory and Sparse Matrix Computation, A. George and J. R. Gilbert and J. W. H. Liu, Eds. New York, NY, USA: Springer-Verlag, 1993.

[30] M. S. Lobo, L. Vandenberghe, S. Boyd, and H. Lebret, "Applications of second-order cone programming," Linear Algebra and its Applications, vol. 284, no. 1-3, pp. 193--228, Nov. 1998.

[31] L. Porkolab and L. Khachiyan, "On the complexity of semidefinite programs," Journal of Glob. Opti., vol. 10, no. 4, pp. 351-365, 1997.

[32] M. V. Ramana, "An exact duality theory for semidefinite programming and its complexity implications," Mathematical Programming, vol. 77, no. 1, pp. 129-162, Apr. 1997.

[33] K. Strunz et al., Benchmark Systems for Network Integration of Renewable and Distributed Energy Resources. CIGRE Task Force C6.04.02.

[34] H. D. Mittelmann, "An independent benchmarking of SDP and SOCP solvers," Math. Program., vol. 95, pp. 407-430, 2003.

[35] W. H. Kersting, "Radial distribution test feeders," in IEEE PES Winter Meeting, vol. 2, Jan. 2001, pp. 908-912.

[36] J. Liu, A. C. Liddell, J. Marecek, and M. Takac, "Hybrid methods in solving alternating-current optimal power flows," IEEE Transactions on Smart Grid, vol. 8, no. 6, pp. 2988-2998, Nov. 2017.

[37] Y. Makarov, D. Hill, and I. Hiskens, "Properties of quadratic equations and their application to power system analysis," International Journal of Electrical Power and Energy Systems, vol. 22, pp. 313-323, 2000. 